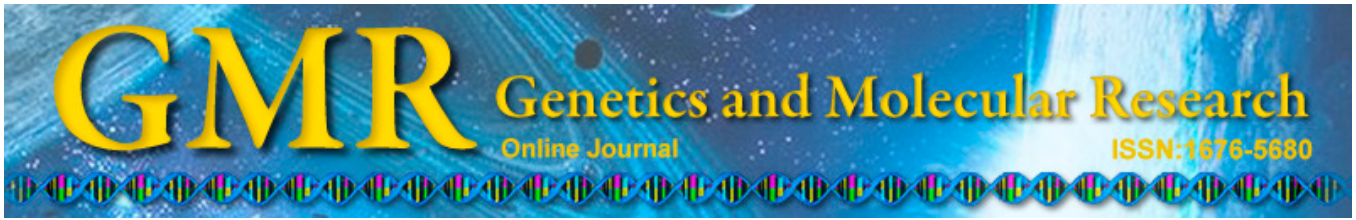

\title{
Sequence characterization and phylogenetic analysis of toll-like receptor $(T L R) 4$ gene in the Tibetan macaque (Macaca thibetana)
}

\author{
Q.X. Dai ${ }^{1,2 *}$, Y.F. Yao ${ }^{1 *}$, Z.C. Qi ${ }^{3 *}$, Y. Huang ${ }^{1}$, Q.Y. Ni ${ }^{1}$, M.W. Zhang ${ }^{1}$ \\ and H.L. $\mathrm{Xu}^{1}$ \\ ${ }^{1}$ College of Animal Science and Technology, Sichuan Agricultural University, \\ Ya'an, China \\ ${ }^{2}$ Forestry College, Sichuan Agricultural University, Ya'an, China \\ ${ }^{3}$ College of Veterinary Medicine, Sichuan Agricultural University, Ya'an, China \\ *These authors contributed equally to this study. \\ Corresponding author: H.L. Xu \\ E-mail: huailxu@yahoo.com \\ Genet. Mol. Res. 14 (1): 1875-1886 (2015) \\ Received January 8, 2014 \\ Accepted October 17, 2014 \\ Published March 13, 2015 \\ DOI http://dx.doi.org/10.4238/2015.March.13.16
}

\begin{abstract}
In this study, the complete coding region sequence of an innate immune-related TLR4 gene was obtained from the Tibetan macaque (Macaca thibetana) genome via PCR and direct sequencing. The sequence had a total length of $2481 \mathrm{bp}$, contained 3 complete exons, and encoded 826 amino acids (AAs); its isoelectric point was 5.703, and the molecular weight was $94.72 \mathrm{kDa}$. The high structure prediction showed that the protein was comprised of one extracellular region, one transmembrane region, and one intracellular region. There were 48 potential functional sites in the protein, including glycosylation, phosphorylation, and acetylation sites. A homology analysis among 9 primate species, including the Tibetan macaque, human, chimpanzee, gibbon, rhesus macaque, cynomolgus monkey, pig-tailed monkey, squirrel monkey, and small-eared galago, showed that the homology
\end{abstract}


of the nucleotide and AA sequences ranged from $60.9-99.5 \%$ and $51.4-$ $99.0 \%$, respectively. Higher variability was identified in the extracellular region of the TLR4 protein, and its variable sites accounted for $88.79 \%$ (AA) of the total variable sites. Additionally, the number of AAs at the $3^{\prime}$ end of the intracellular region was notably different among the primate lineages. The phylogenetic tree based on TLR4 gene exons of 9 primate species showed that the Tibetan macaque clustered with the rhesus macaque, cynomolgus monkey, and pig-tailed monkey; it was most distant from the small-eared galago. This study will provide an important basis for further study on the expression, regulation, and polymorphism of the TLR 4 gene and the relationship between polymorphisms and host disease susceptibility.

Key words: Innate immunity; Macaca thibetana; Phylogenetic analysis; Toll-like receptor; TLR4 gene

\section{INTRODUCTION}

The Tibetan macaque (Macaca thibetana), also known as the Sichuan shorttailed monkey and Daqing Monkey, has a broad distribution in China, mainly in Sichuan, Guizhou, northeastern Yunnan, Hunan, northeastern Guangxi and Guangdong, Fujian, Jiangxi, Zhejiang, and Anhui (Jiang and Wang, 1996). There is also a small population distributed throughout India (Kumar et al., 2005). In recent years, due to the excessive interference of human activities such as the deforestation, reclamation, and poaching, the number of wild Tibetan macaques has drastically reduced and its habitat has been severely fragmented. Currently, the Tibetan macaque is considered an endangered species. It is listed in Appendix II of the Convention on International Trade in Endangered Species of Wild Fauna and Flora (CITES), categorized as "Near Threatened" in the International Union for Conservation of Nature (IUCN) Red List of Endangered Animals, and listed as a second-class protection animal under Chinese wildlife protection laws. Tibetan and rhesus macaques (Macaca mulatta), commonly used as experimental animals, belong to the same genus (Macaca) and have a close genetic relationship. Moreover, the Tibetan macaque has several advantages; it has a longer life span, larger body size, is docile and easy to tame, and has high similarity to the morphology and physiology of humans. Thereby, it has great potential value in biomedical studies such as ophthalmology, obesity, metabolic disease, infectious disease, and organ transplantation (Liu et al., 2011; Ling et al., 2012; Yi et al., 2012). Recently, studies on the Tibetan macaque have primarily focused on its ethology (Zhao, 1997; Li et al., 2007; Xia et al., 2012, 2013), rearing, and disease prevention (Zhong et al., 2010; Wu et al., 2013). In molecular genetics, only a small number of studies on mitochondrial DNA (Li et al., 2008, 2009; Yao et al., 2013a; Zhong et al., 2013), microsatellite DNA (Jia et al., 2011, 2012), and immunity genes (Li et al., 2012; Yao et al., 2013b; Liu et al., 2013) have been reported.

Toll-like receptors (TLRs) are among the most important pattern recognition receptors (PRRs) because they can recognize and respond to pathogen-associated molecular patterns (PAMPs) of bacterium, viruses, and parasites (Akira et al., 2006). TLRs have a wide distribution in organisms; they are expressed in the intestinal epithelium, respiratory epithelial cells, 
endothelial cells, dendritic cells, rat spleen cells, and myocardial cells (Kokkinopoulos et al., 2005; Zhang et al., 2005). TLR4 is an important member of the TLR gene family. It is able to specifically recognize the lipopolysaccharides (LPSs) on the surface of Gramnegative bacteria and is the major receptor of the natural immune system for pathogenic microorganism recognition. Hence, TLR4 is a bridge linking the innate and adaptive immunities. In particular, it plays an important role in congenital innate immunity (Chow et al., 1999; Beutler, 2005). It has been shown that a mutation of the TLR4 gene can change the signal transduction of its coding protein and affect the resistance or susceptibility of the body to various diseases. Mutations of the human TLR 4 gene are able to cause a reduction in response to the LPS of its host, which is associated with the pathogenesis of uterine endometriosis, systemic lupus erythematosus (SLE), and type II diabetes (Rallabhandi et al., 2006; Buraczynska et al., 2009; Verma et al., 2010; Latha et al., 2011). In domestic mammals, it has been correlated with inflammatory bowel disease in dogs, infectious bovine keratitis, and rabbit digestive disorders (Kathrani et al., 2010; Kataria et al., 2011; Zhang et al., 2011). In birds, it has been correlated with susceptibility to salmonella (Leveque et al., 2003). In this study, we sequenced the complete protein coding sequence of an innate immune-related TLR4 gene in the Tibetan macaque via cloning and sequencing technology. In addition, we analyzed the characteristics of its nucleotide and amino acid (AA) sequences and its molecular evolutionary pattern in primates. This study will provide an important theoretical basis for the further study of the structure, function, genetic variation, and relationship to disease susceptibility of the TLR4 gene.

\section{MATERIAL AND METHODS}

\section{Samples}

DNA samples were isolated from the muscle tissue of 2 naturally deceased Tibetan macaque individuals in the wild by using the conventional phenol-chloroform method (Sambrook, 1989) and stored in a $-70^{\circ} \mathrm{C}$ ultra-low temperature freezer for later use at the Wildlife Conservation Laboratory of Sichuan Agricultural University, China. All samples were collected in strict compliance with the Chinese Wildlife Conservation Act and the provisions of the Sichuan Agricultural University Ethics Committee. Materials such as the pMD18-T vector, LaTaq DNA polymerase, and Escherichia coli DH5a were bought from TaKaRa Biotechnology Co. Ltd. (Dalian, China). The PCR gel extraction kit and recombinant plasmid extraction kit were obtained from Shanghai Biological Engineering Technology Services Co. Ltd. (Shanghai, China).

\section{Polymerase chain reaction (PCR)}

According to the complete sequence (including exons and introns) of the TLR4 genes in the human, rhesus macaque, and cynomolgus monkey (Macaca fascicularis) published by GenBank, 3 pairs of PCR primers were designed using the Primer 5.0 software based on relatively conserved regions among the 3 species (Table 1). All primers were synthesized via Shanghai Biological Engineering Technology Services Co. Ltd. 


\begin{tabular}{|c|c|c|c|}
\hline Name & Sequence & Temperature $\left({ }^{\circ} \mathrm{C}\right)$ & Region \\
\hline TLR4-1F & 5'- TGCTGTTTCTTTAGCCACTGGTCTG -3' & 65 & Exon 1 \\
\hline TLR4-1R & 5'- AAGCAGTCTGTTTATTCTCTGGTGA -3' & & \\
\hline TLR4-2F & 5'- CTGCCATTTTATCACAGAGGTTAGA -3' & 63 & Exon 2 \\
\hline TLR4-2R & 5'- TCCACTCCAATGATGAAATGATAGG -3' & & \\
\hline TLR4-3F & 5'- GTCTTTGACCCATCACATCCCGTA -3' & 63 & Exon 3 \\
\hline TLR4-3R & 5'- GTCATAGGTTCCTTGGCTGAGTTG -3' & & \\
\hline
\end{tabular}

Using Tibetan macaque genomic DNA as a template, PCR was performed with a total reaction volume of $20 \mu \mathrm{L}$. This reaction system included $2 \mu \mathrm{L}$ genomic DNA $(30 \mathrm{ng} / \mu \mathrm{L}), 1.6$ $\mu \mathrm{L}$ dNTPs $(2.5 \mathrm{mM}), 1.2 \mu \mathrm{L} \mathrm{MgCl}_{2}(25 \mathrm{mM}), 0.5 \mu \mathrm{L}$ forward primer $(10 \mu \mathrm{M}), 0.5 \mu \mathrm{L}$ reverse primer $(10 \mu \mathrm{M}), 0.5 \mu \mathrm{L}$ Taq DNA Polymerase $(2.5 \mathrm{U} / \mu \mathrm{L}), 14.2 \mu \mathrm{LddH}_{2} \mathrm{O}$. The PCR reaction was as follows: initial denaturation at $94^{\circ} \mathrm{C}$ for $5 \mathrm{~min} ; 35$ cycles of denaturation at $94^{\circ} \mathrm{C}$ for $50 \mathrm{~s}, 63^{\circ} / 65^{\circ} \mathrm{C}$ for $40 \mathrm{~s}$, and $72^{\circ} \mathrm{C}$ for $2 \mathrm{~min}$; followed by a final extension at $72^{\circ} \mathrm{C}$ for $10 \mathrm{~min}$; and then stored at $4^{\circ} \mathrm{C}$. PCR products were observed via $0.8 \%$ agarose gel electrophoresis.

\section{Purifying and sequencing of PCR products}

PCR products were electrophoresed on the $1.2 \%$ agarose gel and then purified and recycled by using a gel extraction kit; the concentration and purity were estimated on the UV transmission stage and finally sequenced via Shanghai Biological Engineering Technology Services Co. Ltd.

\section{Data analysis}

To ensure that the sequences were correct, the obtained sequences were proofread and edited by the Chromosome 1.62 software and SeqMan program (DNASTAR Inc., 1996) of the DNASTAR software package. In this study, a homology blast search was conducted on the National Center for Biotechnology Information (NCBI) website. The base composition and homology analysis of the TLR4 gene coding region sequence were performed using the BioEdit Software. The online protein structure analysis software of simple modular architecture research tool (SMART) (http://smart.Embl-heidelberg.de/) was employed in the prediction of the Tibetan macaque TLR4 protein structure. The DNAMAN was used for the prediction and hydrophobicity analysis of the protein, and the Kyte and Doolittle algorithm was applied for the hydrophobicity analysis (Kyte and Doolittle, 1982). Glycosylation and phosphorylation sites of the Tibetan macaque TLR4 protein were performed by using the ExPASy Proteomics Server Software (http://au.expasy.org), and the prediction of the transmembrane domain was completed by using the TMHMM program (http://www.cbs.dtu.dk/ services/TMHMM). The analysis of sequence characteristics such as base pair differences between sequences, variation sites, parsimony-informative sites, and base compositions were performed using the MEGA 5.05 software (Tamura et al., 2011). The phylogenetic tree was constructed by the Kimura two-parameter model, based on the neighbor-joining (NJ) method with bootstrap test of phylogeny following 1000 replications. The TLR4 gene sequences of 8 other primate species were downloaded from the GenBank and used in this analysis, including Homo sapiens (DQ018109), Pan troglodytes (NM_001144863), Nomascus leucogenys 
(XM_003264057), Macaca mulatta (NM001037092), Macaca fascicularis (AB445643), Macaca nemestrina (GU9696914), Saimiri boliviensis (XM003925187), and Otolemur garnettii (XM003785249).

\section{RESULTS}

\section{Sequence characteristics}

The complete protein coding sequence (CDS) of the Tibetan macaque TLR4 gene (length, $2481 \mathrm{bp}$ ) was obtained by proofreading, sequence assembly, and homology comparison after sequencing; it encodes $826 \mathrm{AAs}$, except for terminator codons. In comparison to the 8 other primate species (i.e., human, chimpanzee, gibbon, rhesus macaque, cynomolgus monkey, pig-tailed monkey, squirrel monkey, and small-eared galago), the length of the Tibetan macaque TLR4 gene CDS was identical to that of the rhesus macaque, cynomolgus monkey, and pig-tailed monkey, which also belong to the genus Macaca but showed significant differences at the 3 ' end from animals

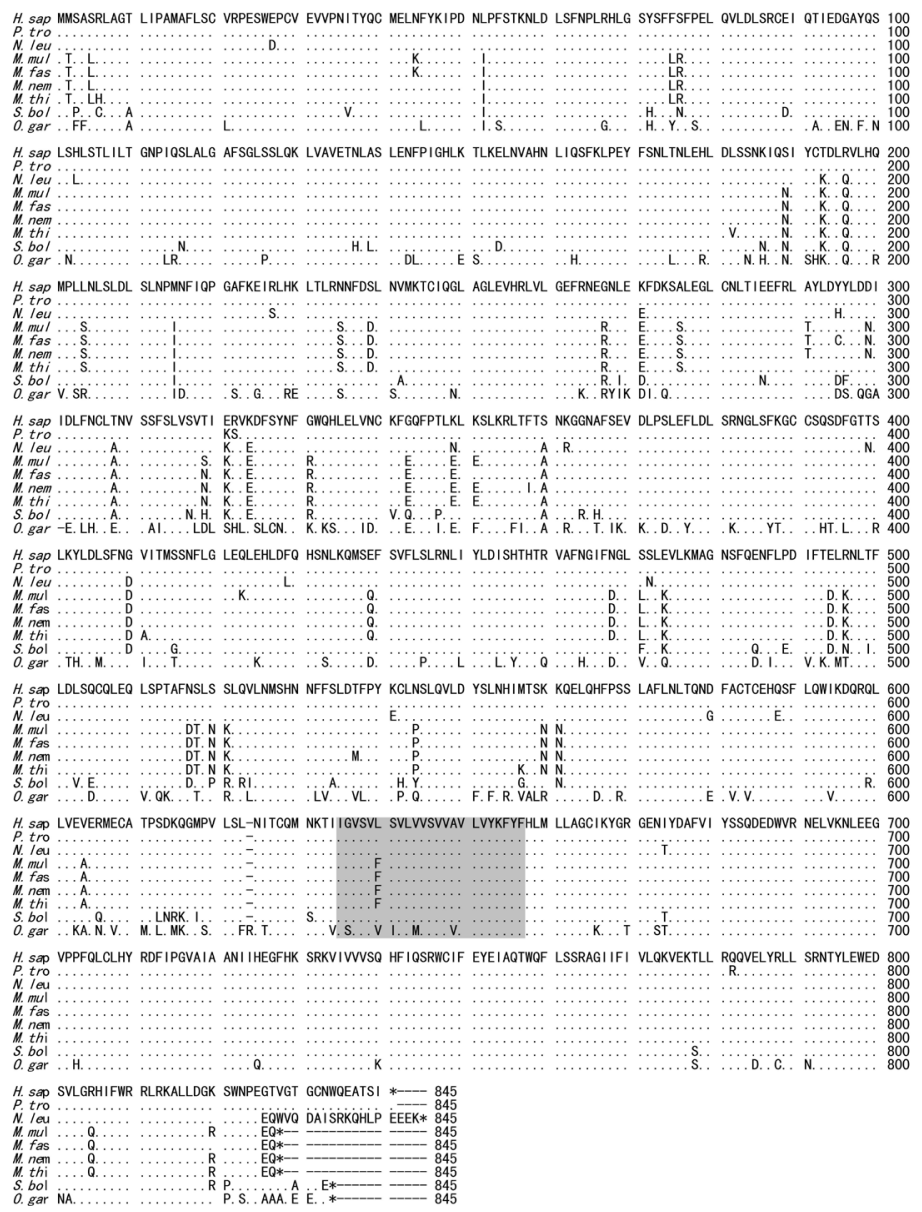

Figure 1. Putative amino acid sequence of the Tibetan-macaque TLR4 gene. Asterisk (*) indicates the stop codon. 
belonging to other genera (Figure 1). For instance, the CDS lengths in the human and chimpanzee were $2520 \mathrm{bp}, 39$ nucleotides (12 AAs) longer than those of Macaca animals at the 3' end; that of the gibbon was the longest in length (2532 bp), which was 51 nucleotides (16 AAs) longer than those of Macaca animals at the 3' end; that of the squirrel and small-eared galago were $2499 \mathrm{bp}, 18$ nucleotides (5 AAs) longer than those of Macaca animals at the 3 ' end. With regard to nucleotide composition, the average contents of $\mathrm{A}, \mathrm{T}, \mathrm{G}$, and $\mathrm{C}$ in the Tibetan macaque TLR4 gene were 27.61, $29.63,20.84$, and $21.98 \%$, respectively, and the $\mathrm{A}+\mathrm{T}(57.23 \%)$ content was higher than that of the $\mathrm{G}+\mathrm{C}(42.77 \%)$ content, which shows that the $\mathrm{A}+\mathrm{T}$ content was higher than that of the $\mathrm{G}+$ $\mathrm{C}$ content for the CDS of the Tibetan macaque TLR4 gene, thus exhibiting an obvious base bias.

\section{Homology analysis of sequences}

The nucleotide or AA sequence similarities of the Tibetan macaque TLR 4 gene with the orthologous sequences of 8 other primate species were analyzed in this study (Table 2). These 8 included the human, chimpanzee, gibbon, rhesus macaque, cynomolgus monkey, pig-tailed

Table 2. Identity of nucleotide and amino acid sequences for 8 species.

\begin{tabular}{|c|c|c|c|c|c|c|c|c|c|}
\hline & H. sap & P. tro & N. leu & M. mul & M. fas & M. nem & M. thi & S. bol & O. gar \\
\hline H. sap & & 0.996 & 0.951 & 0.948 & 0.948 & 0.948 & 0.948 & 0.904 & 0.519 \\
\hline P. tro & 0.994 & & 0.951 & 0.949 & 0.949 & 0.948 & 0.949 & 0.905 & 0.517 \\
\hline N. leu & 0.966 & 0.966 & & 0.943 & 0.943 & 0.942 & 0.943 & 0.892 & 0.512 \\
\hline M. mul & 0.926 & 0.926 & 0.920 & & 0.993 & 0.996 & 0.989 & 0.903 & 0.514 \\
\hline M. fas & 0.928 & 0.928 & 0.924 & 0.995 & & 0.995 & 0.993 & 0.905 & 0.515 \\
\hline M. nem & 0.926 & 0.926 & 0.920 & 0.993 & 0.992 & & 0.990 & 0.903 & 0.515 \\
\hline M. thi & 0.924 & 0.926 & 0.920 & 0.995 & 0.987 & 0.995 & & 0.906 & 0.514 \\
\hline S. bol & 0.928 & 0.929 & 0.916 & 0.923 & 0.923 & 0.923 & 0.924 & & 0.522 \\
\hline O. gar & 0.612 & 0.612 & 0.606 & 0.608 & 0.609 & 0.610 & 0.609 & 0.617 & \\
\hline
\end{tabular}

Upper triangle shows the identity of the DNA sequences, lower triangle shows the identity of amino acid sequences; species names are abbreviations of the scientific names: H. sapiens, P. troglodytes, $N$. leucogenys, M. mulatta, $M$. fascicularis, M. nemestrina, S. boliviensis and O. garnettii. (e.g., M. thi is an abbreviation of M. thibetana).

monkey, squirrel monkey, and small-eared galago. The results showed that the identities of the nucleotide sequences were $94.8,94.9,94.3,99.5,99.3,99.5,92.4$, and $60.9 \%$, respectively, and the identities of the deduced AA sequences were 92.4, 92.6, 92.0, 98.9, 98.7, 99.0, 90.6, and $51.4 \%$, respectively. The results of the comparison showed that the TLR4 gene was highly conservative across species. After further aligning the nucleotide and AA sequences of the TLR4 gene among the 9 species, 505 nucleotide variable sites were detected in the alignment of the coding nucleotide sequence (on the basis of the Tibetan macaque nucleotide sequence of $2481 \mathrm{bp}$ ), accounting for $20.35 \%$ of the total number of all sites. There were 109 parsimonyinformative sites, accounting for $21.58 \%$ of the total number of all variable sites; and 396 singleton sites, accounting for $78.42 \%$ of the total number of all variable sites. Moreover, a comparison of the deduced AA sequence (on the basis of the Tibetan macaque AA sequence of $826 \mathrm{AAs}$ ), $232 \mathrm{AA}$ variable sites were detected, accounting for $28.09 \%$ of the total number of all sites. There were 54 parsimony-informative sites, accounting for $10.69 \%$ of all variable sites; and 178 singleton sites, accounting for $35.25 \%$ of the total number of all variable sites. In addition, when the extracellular, transmembrane, and intracellular regions were analyzed, it was found that the variability of the extracellular region was greater than that of the other regions (i.e., the transmembrane and intracellular regions) (Table 3). 
Table 3. Variable sites of the TLR4-gene DNA/AA sequences in 9 primate species.

\begin{tabular}{lcccc}
\hline Sequence & Complete CDS & Extracellular & Transmembrane & Intracellular \\
\hline DNA (2481) & 505 & $436(86.34)$ & $13(2.57)$ & $56(11.09)$ \\
AA (826) & 232 & $206(88.79)$ & $7(3.02)$ & $20(8.62)$ \\
\hline
\end{tabular}

In the sequence column, the number in parentheses indicates total sequence length; in all other columns, the numbers that are and are not in parentheses, indicate variable sites in each region, but percentage values are in parentheses and the percentage of variable sites in each region in comparison to total variable sites (DNA, 505; AA, 232), respectively.

\section{Protein characteristics}

The TLR4 protein prediction analysis was performed by using the Proteomics Server software. The results showed that the protein had a molecular weight of $94.72 \mathrm{kDa}$, a PI of 5.703, a charge of 18.614 at $\mathrm{pH} 7.0$; it contained 89 AA residues with negative charges (aspartate and glutamic acid), 67 AA residues with positive charges (lysine and arginine), and the other AAs were neutral; the polarity analysis of the AAs showed that the protein contained 252 polar AA residues (i.e., asparagine, cysteine, glutamine, serine, threonine, and tyrosine) and 322 nonpolar AA residues (i.e., alanine, isoleucine, leucine, phenylalanine, tryptophan, and valine). This protein has 3 types of 48 potential functional sites, including one threonine glycosylation site (611TPS), one serine glycosylation site (622SLN), $10 \mathrm{~N}$-glycosylation sites (i.e., 35NITY, 173NLTN, 205NLSL, 282NLTI, 309NVSS, 497NLTF, 526NMSH, 575NLTQ, 624NITC, and 630NKTI); 22 serine phosphorylation sites, 7 tyrosine phosphorylation sites, 5 threonine phosphorylation sites, one threonine acetylation site (2TAS), and one threonine acetylation site (3SAL). The online TLR4 protein structure analysis of 3 primate species (i.e., human, Tibetan macaque, and rhesus macaque) by SMART (Figure 2) showed that the Tibetan macaque TLR4 protein was a transmembrane protein comprised of one extracellular region (AA1-633, 633 AAs), one transmembrane region (AA634-656, 23 AAs), and one intracellular region (AA657-826, 170 AAs). The extracellular region contained 12 leucine-rich repeats

\section{Extracellular}
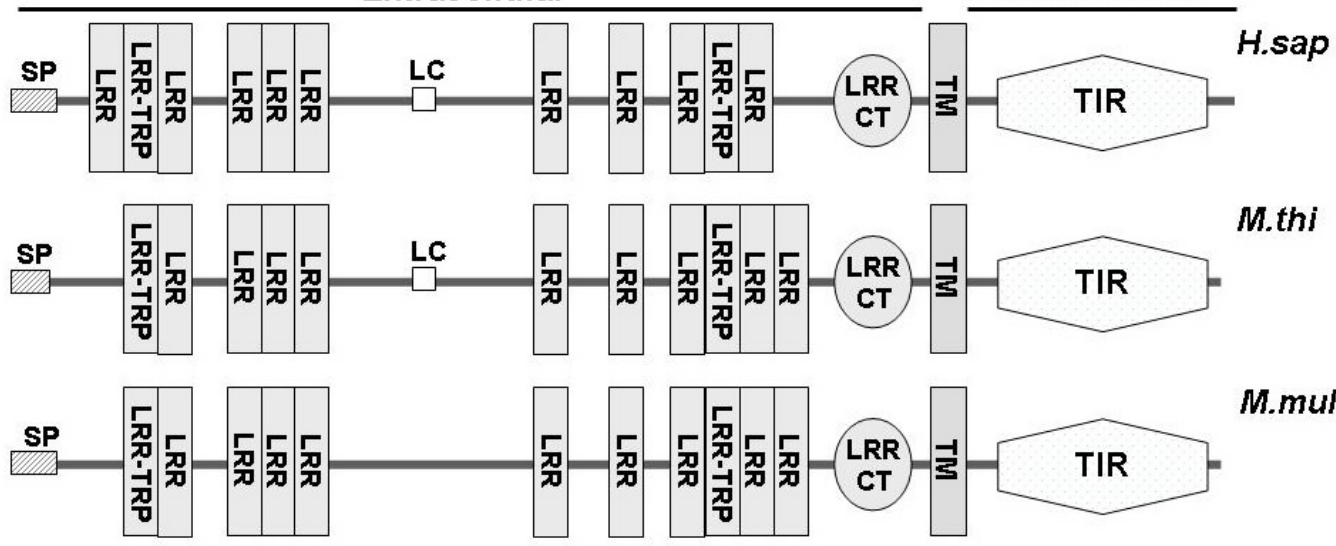

Figure 2. Structural domains of the TLR4 protein among 3 primate species. LRR, leucine-rich repeat; TM, transmembrane region; TIR, Toll/IL-1 like receptor homologous region; SP, signal peptide; LC, low complexity region. 
(LRRs), and the intracellular region contained one Toll/IL-1 like receptor homologous region (TIR). The Tibetan macaque TLR4 protein structure was almost entirely identical to that of the rhesus macaque but had many differences when compared to that of the human, primarily in the extracellular region. For instance, there were 5 LRRs on the left end and 7 LRRs on the right end in the rhesus macaque TLR4 protein extracellular region; there were 6 LRRs on the left end and 6 LRRs on the right end in that of the human; there was a low complexity region in the middle of the extracellular region for both species. The AA hydrophobicity analysis of the Tibetan macaque TLR4 protein by using the DNAMAN software, in accordance with the default parameters, showed that most regions of the Tibetan macaque TLR4 protein were hydrophobic and only 81 sections were hydrophilous. The number of the hydrophilous AA residues accounted for $43.16 \%$ of the number of the total AA residues (357/827). Taking into account the position of the 81 hydrophilous sections, there were 60 at the extracellular region, 3 at the transmembrane region and 18 at the intracellular region (Figure 3).

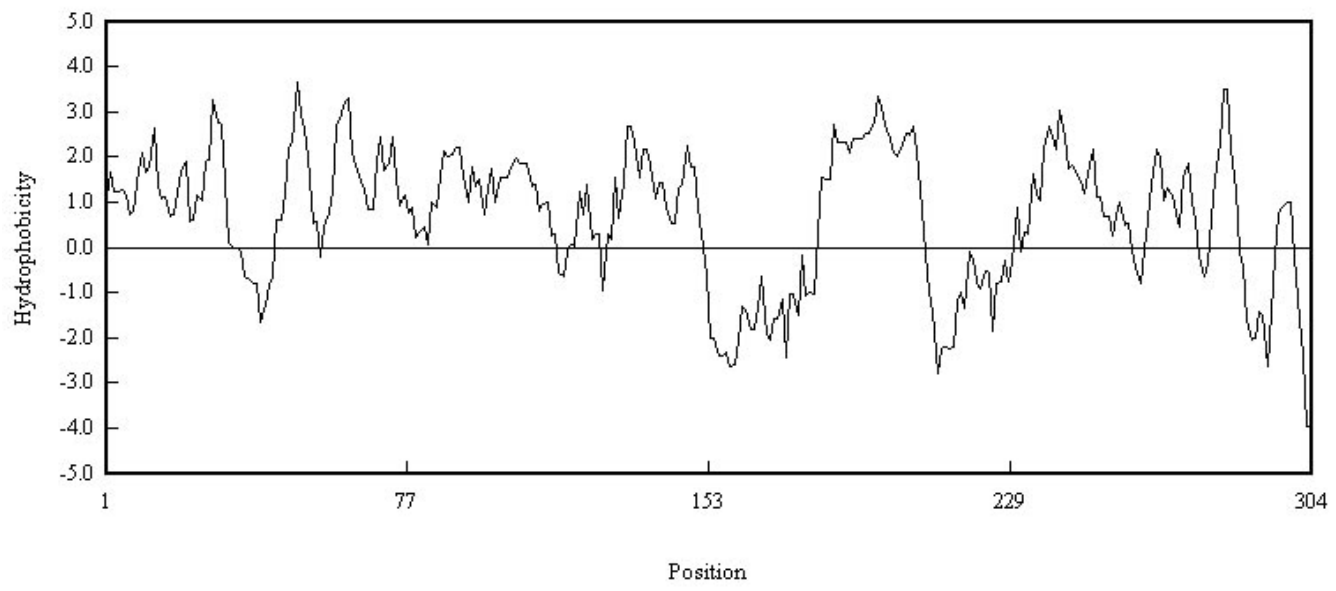

Figure 3. Hydrophobicity plot of the deduced amino acid sequence of the TLR4 gene. Hydrophobic segments are characterized by positive values.

\section{Phylogenetic analysis}

The phylogenetic tree was constructed based on TLR4 gene exons of the Tibetan macaque and the homologous sequence of other primate species, including the human, chimpanzee, gibbon, rhesus macaque, cynomolgus monkey, pig-tailed monkey, squirrel monkey, and small-eared galago (Figure 4). The phylogenetic tree showed that the Tibetan macaque, rhesus macaque, and cynomolgus monkey, which all belong to the same genus (Macaca), constituted a single clade, and the others formed a separate clade. First, the human and chimpanzee formed a sister relationship; they then clustered with the gibbon, 4 Macaca animals, and squirrel monkey; finally, they clustered with the small-eared galago, which belongs to the Prosimii. The Tibetan macaque was most closely related to the other 3 Macaca animals, the more closely related gibbon, chimpanzee, human, and squirrel monkey, in turn, and most distantly related to the small-eared galago. This result is consistent with the results of the homology comparison. 


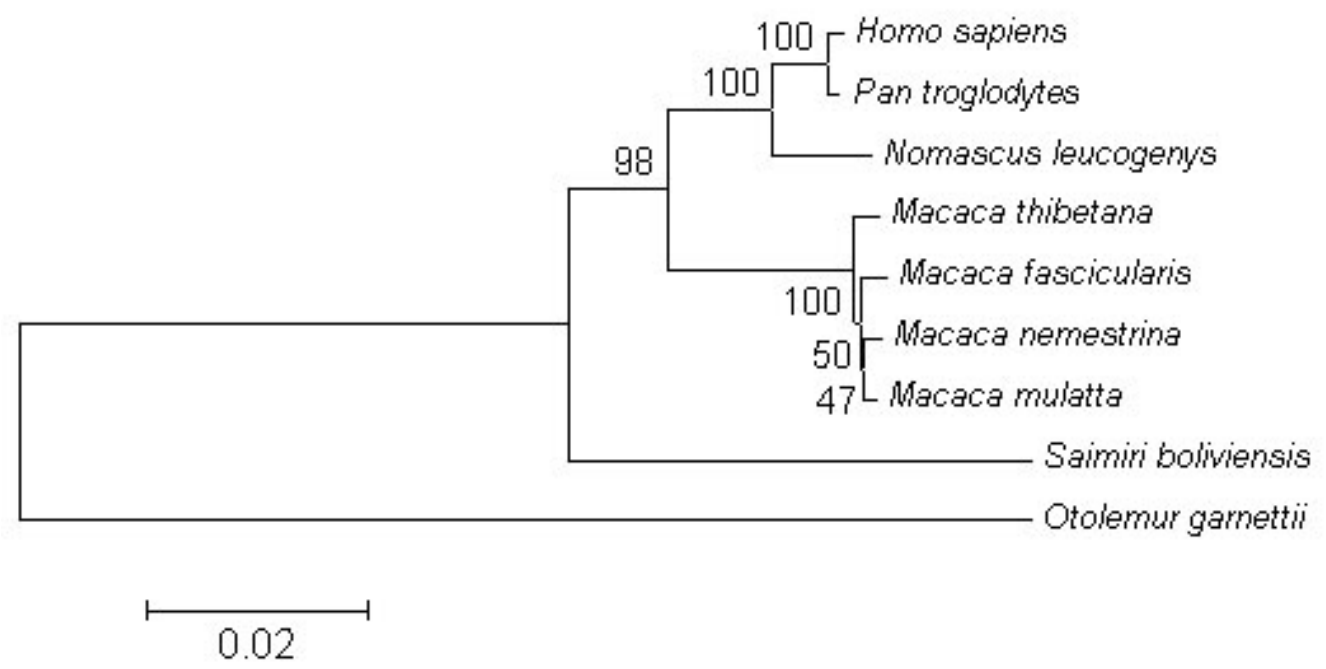

Figure 4. Neighbor joining (NJ) tree of TLR4 gene exons of 9 primate species. Numbers at the nodes indicate a bootstrap value $>50 \%$.

\section{DISCUSSION}

Nonhuman primates have the closest relationship to humans, and most have been widely used in human disease-related biomedical research. Understanding the genetic, especially the immunogenetic, background of nonhuman primates is crucial for identifying applications in biomedical research. TLR4 is an important member of the TLRs, it can specifically recognize the LPS on the surface of Gram-negative bacteria, and it is the major receptor of the natural immune system that recognizes pathogenic microorganisms. In this study, the complete coding sequence of the TLR4 gene was obtained from the Tibetan macaque genome by using PCR and direct sequencing. The sequence has a total length of $2481 \mathrm{bp}, 3$ complete exons, and encodes 826 AAs. The prediction of high protein structure showed that the Tibetan macaque TLR4 protein was a transmembrane protein comprised of one intracellular region, one extracellular region, and one transmembrane region, which is characteristic of other TLRs members (Huang et al., 2012). There was higher homology in the nucleotide and AA sequence between the Tibetan macaque and the 8 other primate species (i.e., human, chimpanzee, gibbon, rhesus macaque, cynomolgus monkey, pig-tailed monkey, squirrel monkey, and smalleared galago), ranging from $60.9-99.5 \%$ to $51.4-99.0 \%$, respectively. In fact, except for the small-eared galago, the nucleotide and AA sequence homologies were both $>90 \%$; in particular, the sequence homologies of the Tibetan macaque, rhesus macaque, and cynomolgus monkey, all belonging to the same genus Macaca, were up to $99 \%$ for nucleotide acids and $98 \%$ for AAs. The results show that the TLR4 gene is highly conserved in primates.

While TLR4 gene has a high degree of similarity among the different primate animals, it also presented a certain degree of variability between the 9 primate animals used in this study. For instance, in the alignment of the coding region nucleotide sequences (on the basis of the Tibetan macaque nucleotide sequence, $2481 \mathrm{bp}$ ), a total of 505 nucleotide variable sites were found, accounting for $20.35 \%$ of the total number of sites analyzed. With regard to the 
alignment of the deduced AA sequences (on the basis of the Tibetan macaque AA sequence, 826 AAs), 232 AA variable sites were found, accounting for $28.09 \%$ of the total number of sites analyzed. Further analysis showed that these variable sites were mainly located within the extracellular region (DNA, 86.34\%; AA, 88.79\%), which coincided with the function of the extracellular region because the region plays a role in the specific recognition of a pathogen and its products. The results showed that the notable variability in the extracellular region of the TLR4 gene might be related to differences in pathogen recognition among the different primates. In particular, between human and Macaca animals (i.e., Tibetan and rhesus macaques), the significant difference in the LRR composition pattern within the extracellular region of the TLR4 protein was likely a functional adaptation in the evolutionary process from nonhuman primates to humans. It is clear that human beings encounter distinct pathogenic microorganisms when compared to nonhuman primates. It is worth noting that there were significant differences in the number of $3^{\prime}$ end AAs, although a few interspecific variable sites were observed in the intracellular region (DNA, 11.09\%; AA, 8.62\%). For instance, compared to the 3' end lengths of the Macaca animal TLR4 genes, those of the human and chimpanzee were 39 nucleotides (12 AAs) longer, the gibbon was 51 nucleotides (16 AAs) longer, and both the squirrel monkey and small-eared galago were 18 nucleotides (5 AAs) longer. The major function of the TLR4-protein intracellular region was to mediate the signal transduction and, finally, induce the immune response by providing docking sites to intracellular adaptor proteins (such as MyD88). Thus, the notable difference in the number of AAs of the $3^{\prime}$ end in the TLR4 protein intracellular region between different primates is likely related to their connection to different intracellular adaptor proteins, which ultimately induces different signal transduction pathways (Martin and Wesche, 2002); however, further study is required.

For the 9 primate species assessed in this study, it has been shown that the human is most closely related to the chimpanzee; then, the gibbon, Macaca animals (i.e., Tibetan macaque, rhesus macaque, and cynomolgus monkey), squirrel monkey, and small-eared galago; the human was most distantly related to the small-eared galago (Goodman et al., 1998). In the present study, the topology of our phylogenetic tree based on the TLR 4 gene exons strongly supports this result. However, among the 4 Macaca animals (i.e., Tibetan macaque, rhesus macaque, cynomolgus monkey, and pig-tailed monkey), which are closely related to each other, there was significant difference between our phylogenetic tree and the results obtained on the basis of morphological evidence. For instance, in a study on Macaca evolution (Jiang et al., 1992), the Tibetan and rhesus macaques were closest to the pig-tailed and cynomolgus monkeys, respectively. However, in our study, the rhesus macaque was more closely related to the pig-tailed monkey, which then clustered with the cynomolgus monkey and Tibetan macaque. These results indicate that the phylogenetic tree constructed based on the primate TLR4 gene exons can provide a rough estimate of the phylogenetic relationship among pedigrees with distant relationships (for example, between families) but was unsuitable for judging the phylogenetic relationship among closely related pedigrees (e.g., between species within the same genus).

In conclusion, our results primarily show that the TLR 4 gene is highly conservative among all the primate species assessed in this study, particularly with regard to the genus Macaca. However, high variation was also found in the extracellular region and 3' end of the intracellular region, indicating functional adaptive evolution in primates. This study provides a basis for further study on the expression, regulation, polymorphism of the TLR4 gene and the relationship between the polymorphisms and host disease susceptibility. 


\section{ACKNOWLEDGMENTS}

Research supported by the National Natural Science Foundation of China (\#30970383, \#31370407), Natural Science Foundation of Educational Commission of Sichuan Province of China (\#08ZA076), Academic Leader Training Projects in Sichuan Province of China (\#01320206) and the Innovative Research Team in University of Sichuan Bureau of Education.

\section{REFERENCES}

Akira S, Uematsu S and Takeuchi O (2006). Pathogen recognition and innate immunity. Cell 124: 783-801.

Beutler B (2005). The toll-like receptors: analysis by forward genetic methods. Immunogenetics 57: 385-392.

Buraczynska M, Baranowicz-Gaszczyk I, Tarach J and Ksiazek A (2009). Toll-like receptor 4 gene polymorphism and early onset of diabetic retinopathy in patients with type 2 diabetes. Hum. Immunol. 70: 121-124.

Chow JC, Young DW, Golenbock DT, Christ WJ, et al. (1999). Toll-like receptor 4 mediates lipopolysaccharide-induced signal transduction. J. Biol. Chem. 274: 10689-10692.

Goodman M, Porter CA, Czelusniak J, Page SL, et al. (1998). Toward a phylogenetic classification of Primates based on DNA evidence complemented by fossil evidence. Mol. Phylogenet. Evol. 9: 585-598.

Huang Y, Yao YF, Li Y, Ni QY, et al. (2012). Identification and molecular evolutionary analysis of TLR5 gene from the Tibetan macaque (Macaca thibetana). Genes \& Genomics. 34: 423-428.

Jia XD, Yang BD, Yue BS, Yin HL, et al. (2011). Isolation and characterization of twenty-one polymorphic microsatellite loci in the Tibetan macaque (Macaca thibetana). Genetika 47: 996-999.

Jia XD, Zhang XY, Wang HX, Wang ZK, et al. (2012). Construction of microsatellite-enriched library and isolation of microsatellite markers in Macaca thibetana. Sichuan J. Zool. 31: 39-44.

Jiang X and Wang Y (1996). Taxonomy and distribution of Tibetan macaque. Zool. Res. 17: 361-369.

Jiang XL, Wang YX, Ma SL and He YH (1992). Cluster analysis and evolutionary relationships of Chinese macaques. Acta Anthropologica Sinica 11: 184-191.

Kathrani A, House A, Catchpole B, Murphy A, et al. (2010). Polymorphisms in the TLR4 and TLR5 gene are significantly associated with inflammatory bowel disease in German shepherd dogs. PLoS One 5: el5740.

Kataria RS, Tait RG Jr, Kumar D, Ortega MA, et al. (2011). Association of toll-like receptor four single nucleotide polymorphisms with incidence of infectious bovine keratoconjunctivitis (IBK) in cattle. Immunogenetics 63: 115119.

Kokkinopoulos I, Jordan WJ and Ritter MA (2005). Toll-like receptor mRNA expression patterns in human dendritic cells and monocytes. Mol. Immunol. 42: 957-968.

Kumar RS, Mishra C and Sinha A. (2005). Discovery of the Tibetan macaque Macaca thibetana in Arunachal Pradesh, India. Curr. Sci. 88: 1387-1388.

Kyte J and Doolittle RF (1982). A simple method for displaying the hydropathic character of a protein. J. Mol. Biol. 157: 105-132.

Latha M, Vaidya S, Mowa S, Chava S, et al. (2011). Molecular pathogenesis of endometriosis; Toll-like receptor-4 A896G (D299G) polymorphism: a novel explanation. Genet. Test Mol. Biomarkers. 15: 181-184.

Leveque G, Forgetta V, Morroll S, Smith AL, et al. (2003). Allelic variation in TLR4 is linked to susceptibility to Salmonella enterica serovar Typhimurium infection in chickens. Infect. Immun. 71: 1116-1124.

Li D, Fan L, Ran J, Yin H, et al. (2008). Genetic diversity analysis of Macaca thibetana based on mitochondrial DNA control region sequences. Mitochondrial DNA 19: 446-452.

Li D, Fan L, Zeng B, Yin H, et al. (2009). The complete mitochondrial genome of Macaca thibetana and a novel nuclear mitochondrial pseudogene. Gene 429: 31-36.

Li J, Yin H and Zhou L (2007). Non-reproductive copulation behavior among Tibetan macaques (Macaca thibetana) at Huangshan, China. Primates. 48: 64-72.

Li JY, Yao YF, Zhou L and Xu HL (2012). Polymorphic analysis of Mhc-DPB1 gene exon 2 in Tibetan macaques (Macaca thibetana). Yi Chuan 34: 1417-1426.

Ling J, Yao F and Zhong H (2012). Effect of dietary protein levels on juvenile Tibetan macaque (Macaca thibetana). Chinese Journal of Comparative Medicine 22: 36-40.

Liu G, Zeng T, Yu W, Yan N, et al. (2011). Characterization of intraocular pressure responses of the Tibetan monkey (Macaca thibetana). Mol. Vis. 17: 1405-1413.

Liu W, Yao YF, Zhou L, Ni QY, et al. (2013). Evolutionary analysis of the short-type peptidoglycan-recognition protein 
gene (PGLYRP1) in primates. Genet. Mol. Res. 12: 453-462.

Martin MU and Wesche H. (2002). Summary and comparison of the signaling mechanisms of the Toll/interleukin-1 receptor family. Biochim. Biophys. Acta 1592: 265-280.

Rallabhandi P, Bell J, Boukhvalova MS, Medvedev A, et al. (2006). Analysis of TLR4 variants: new insights into TLR4/ MD-2/CD14 stoichiometry, structure, and signaling. J. Immunol. 177: 322-332.

Sambrook J, Fritsch EF and Maniatis T (1989). Molecular cloning: a laboratory manual. Cold Spring Harbor Laboratory Press, New York.

Tamura K, Peterson D, Peterson N, Stecher G et al. (2011). MEGA5: molecular evolutionary genetics analysis using maximum likelihood, evolutionary distance, and maximum parsimony methods. Mol. Biol. Evol. 28: 2731-2739.

Verma A, Prasad KN, Gupta RK, Singh AK, et al. (2010). Toll-like receptor 4 gene polymorphism and its association with symptomatic neurocysticercosis. J. Infect. Dis. 202: 1219-1225.

Wu D, Jiang WY, Yang F, Wei SY, et al. (2013). Somatometric measurements, and clinical chemistry and hematology parameters in Tibetan macaque (Macaca thibetana). J. Med. Primatol. 42: 318-324.

Xia D, Li J, Matheson MD, Sun L, et al. (2012). First occurrence of twins in provisioned free-ranging Tibetan macaques (Macaca thibetana) at Huangshan, China. Primates 53: 1-5.

Xia DP, Li JH, Garber PA, Matheson MD, et al. (2013). Grooming reciprocity in male Tibetan macaques. Am. J. Primatol. 75: 1009-1020.

Yao Y, Zhong L, Liu B, Li J, et al. (2013a). Genetic variation between two Tibetan macaque (Macaca thibetana) populations in the eastern China based on mitochondrial DNA control region sequences. Mitochondrial DNA 24: 267-275.

Yao YF, Zhao JJ, Dai QX, Li JY, et al. (2013b). Identification and characterization of the major histocompatibility complex class II DQB (MhcMath-DQB1) alleles in Tibetan macaques (Macaca thibetana). Tissue Antigens 82: 113-121.

Yi Y, Zeng T, Zhou L, Cai SP, et al. (2012). Experimental Tibetan monkey domestication and its application for intraocular pressure measurement. Int. J. Ophthalmol. 5: 277-280.

Zhang GW, Wang HZ, Chen SY, Li ZC, et al. (2011). A reduced incidence of digestive disorders in rabbits is associated with allelic diversity at the TLR4 locus. Vet. Immunol. Immunopathol. 144: 482-486.

Zhang X, Shan P, Qureshi S, Homer R, et a1. (2005). TLR4 deficiency confers susceptibility to lethal oxidant lung injury. J. Immunol. 175: 4834-4838.

Zhao QK (1997). Intergroup interactions in Tibetan macaques at Mt. Emei, China. Am. J. Phys. Anthropol. 104: 459-470.

Zhong ZD, Xu YZ, Yao F, Wei JJ, et al. (2010). Pathological observation on Macaca thibetana with secondary infection cause by diarrhea. Laboratory Animal and Comparative Medicine. 6: 432-435.

Zhong LJ, Zhang MW, Yao YF, Ni QY, et al. (2013). Genetic diversity of two Tibetan macaque (Macaca thibetana) populations from Guizhou and Yunnan in China based on mitochondrial DNA D-loop sequences. Genes Genom. 35: $205-214$ 